\title{
A framework for developing prediabetes self-care application
}

\begin{abstract}
Preventing diabetes at the prediabetes stage is a crucial step to take as the damages done by diabetes is non-reversible. In the recent years, the number of diabetics has been increasing tremendously, which is predominantly due to unhealthy lifestyle and diet. To prevent diabetes, human behaviour needs to be altered: a new positive habit should replace an old habit. Self-care applications have great potential in fostering diabetes prevention. However, existing solutions mostly do not follow clinical guidelines, do not incorporate user expectations, are aimed to deliver a specific functionality (such as weight management), are not designed based on behavioural change theories, and therefore they are not designed for long term use. Integrating health behavioural change theories and the prediabetics' requirements into the development of self-care applications can empower consumers to better care of their own health. In this paper, we propose a prediabetes self-care application design framework, which incorporates behavioural change theories, and meets requirements of prediabetics. The framework was validated by a pool of health-related experts from various fields. Results indicate that the framework comprised necessary functions that would enable prediabetes to prevent diabetes. The framework would aid developers to design novel prediabetes applications that would enable users to take greater responsibility towards their health, which in return helps to save medical cost.
\end{abstract}

Keyword: Diabetes prevention; Behavioural change theories; Health informatics; Behaviour change techniques; Web technology; Mobile application 